Supplement of Hydrol. Earth Syst. Sci., 25, 2843-2860, 2021

https://doi.org/10.5194/hess-25-2843-2021-supplement

(c) Author(s) 2021. CC BY 4.0 License.

(c) (i)

Supplement of

\title{
Urban surface water flood modelling - a comprehensive review of current models and future challenges
}

\section{Kaihua Guo et al.}

Correspondence to: Mingfu Guan (mfguan@hku.hk)

The copyright of individual parts of the supplement might differ from the article licence. 
Table S1. Literature pool

\begin{tabular}{|c|c|c|c|c|c|}
\hline No. & Author & Journal/ institute & Year & Title & Study area \\
\hline 1 & $\begin{array}{l}\text { Djordjevic S. } \\
\text { et.al }\end{array}$ & $\begin{array}{l}\text { Water Science and } \\
\text { Technology }\end{array}$ & 1999 & An approach to simulation of dual drainage & \multirow{5}{*}{$\begin{array}{l}\text { Drainage } \\
\text { network models }\end{array}$} \\
\hline 2 & Mark O. et.al & Journal of Hydrology & 2004 & $\begin{array}{l}\text { Potential and limitations of } 1 \mathrm{D} \text { modelling of urban } \\
\text { flooding }\end{array}$ & \\
\hline 3 & $\begin{array}{l}\text { Schmitt T. } \\
\text { G. et.al }\end{array}$ & Journal of Hydrology. & 2004 & $\begin{array}{l}\text { Analysis and modelling of flooding in urban drainage } \\
\text { systems }\end{array}$ & \\
\hline 4 & $\begin{array}{l}\text { Djordjevic S. } \\
\text { et.al }\end{array}$ & $\begin{array}{l}\text { Water Science \& } \\
\text { Technology }\end{array}$ & 2005 & $\begin{array}{l}\text { SIPSON-simulation of interaction between pipe flow and } \\
\text { surface overland flow in networks }\end{array}$ & \\
\hline 5 & $\begin{array}{l}\text { Rossman } \\
\text { L.A. }\end{array}$ & $\begin{array}{l}\text { US Environmental } \\
\text { Protection Agency }\end{array}$ & 2010 & Storm water management model user's manual, version 5. & \\
\hline 6 & $\begin{array}{l}\text { Bradbrook } \\
\text { K.F. et.al }\end{array}$ & $\begin{array}{l}\text { International Journal of } \\
\text { River Basin Management }\end{array}$ & 2004 & $\begin{array}{l}\text { Two dimensional diffusion wave modelling of flood } \\
\text { inundation using a simplified channel representation. }\end{array}$ & \multirow{12}{*}{ Simplified 2D shallow water models } \\
\hline 7 & Rao P & $\begin{array}{l}\text { Environmental Modelling } \\
\text { and Software }\end{array}$ & 2005 & $\begin{array}{l}\text { A parallel RMA2 model for simulating large-scale free } \\
\text { surface flows }\end{array}$ & \\
\hline 8 & Yu D. et.al & $\begin{array}{l}\text { Hydrological Processes: } \\
\text { An International Journal }\end{array}$ & 2006 & $\begin{array}{l}\text { Urban fluvial flood modelling using a two-dimensional } \\
\text { diffusion-wave treatment, part } 1: \text { mesh resolution effects }\end{array}$ & \\
\hline 9 & $\begin{array}{l}\text { Hunter N.M. } \\
\text { et.al }\end{array}$ & Geomorphology & 2007 & $\begin{array}{l}\text { Simple spatially-distributed models for predicting flood } \\
\text { inundation: A review }\end{array}$ & \\
\hline 10 & Yu D & $\begin{array}{l}\text { Environmental Modelling } \\
\text { and Software }\end{array}$ & 2010 & $\begin{array}{l}\text { Parallelization of a two-dimensional flood inundation } \\
\text { model based on domain decomposition }\end{array}$ & \\
\hline 11 & Bates P. et.al & Journal of Hydrology & 2010 & $\begin{array}{l}\text { A simple inertial formulation of the shallow water } \\
\text { equations for efficient two-dimensional flood inundation } \\
\text { modelling }\end{array}$ & \\
\hline 12 & $\begin{array}{l}\text { Chen A.S. } \\
\text { et.al }\end{array}$ & Journal of Hydrology & 2012 & $\begin{array}{l}\text { A coarse-grid approach to representing building blockage } \\
\text { effects in 2D urban flood modelling }\end{array}$ & \\
\hline 13 & $\begin{array}{l}\text { Almeida G. } \\
\text { et.al }\end{array}$ & $\begin{array}{l}\text { Water Resources } \\
\text { Research }\end{array}$ & 2013 & $\begin{array}{l}\text { Applicability of the local inertial approximation of the } \\
\text { shallow water equations to flood modeling }\end{array}$ & \\
\hline 14 & Zhang H & Newcastle University & 2014 & $\begin{array}{l}\text { Urban flood simulation by coupling a hydrodynamic } \\
\text { model with a hydrological model. }\end{array}$ & \\
\hline 15 & $\begin{array}{l}\text { Henonin } \mathrm{J} . \\
\text { et.al }\end{array}$ & Urban Water Journal & 2015 & $\begin{array}{l}\text { Citywide multi-grid urban flood modelling: The July } 2012 \\
\text { flood in Beijing }\end{array}$ & \\
\hline 16 & $\begin{array}{l}\text { Leandro J. } \\
\text { et.al }\end{array}$ & $\begin{array}{l}\text { Water Science and } \\
\text { Technology }\end{array}$ & 2016 & $\begin{array}{l}\text { A methodology for linking 2D overland flow models with } \\
\text { the sewer network model SWMM } 5.1 \text { based on dynamic } \\
\text { link libraries }\end{array}$ & \\
\hline 17 & $\begin{array}{l}\text { Sanders B. } \\
\text { et.al }\end{array}$ & $\begin{array}{l}\text { Advances in Water } \\
\text { Resources }\end{array}$ & 2019 & PRIMo: Parallel raster inundation model & \\
\hline
\end{tabular}




\begin{tabular}{|c|c|c|c|c|c|}
\hline 18 & $\begin{array}{l}\text { Huang H. } \\
\text { et.al }\end{array}$ & Hydrology Research & 2019 & $\begin{array}{l}\text { An urban pluvial flood simulation model based on } \\
\text { diffusive wave approximation of shallow water equations }\end{array}$ & \\
\hline 19 & $\begin{array}{l}\text { Audusse E. } \\
\text { et.al }\end{array}$ & $\begin{array}{l}\text { SIAM Journal on } \\
\text { Scientific Computing }\end{array}$ & 2004 & $\begin{array}{l}\text { A fast and stable well-balanced scheme with hydrostatic } \\
\text { reconstruction for shallow water flows. }\end{array}$ & \multirow{9}{*}{ Full 2D shallow water models } \\
\hline 20 & $\begin{array}{l}\text { Liang Q. } \\
\text { et.al }\end{array}$ & $\begin{array}{l}\text { Advances in water } \\
\text { resources }\end{array}$ & 2009 & $\begin{array}{l}\text { Numerical resolution of well-balanced shallow water } \\
\text { equations with complex source terms. }\end{array}$ & \\
\hline 21 & Toro E.F. & $\begin{array}{l}\text { Springer Science \& } \\
\text { Business Media }\end{array}$ & 2013 & $\begin{array}{l}\text { Riemann solvers and numerical methods for fluid } \\
\text { dynamics: a practical introduction. }\end{array}$ & \\
\hline 22 & Kim B. et.al & Journal of Hydrology & 2015 & $\begin{array}{l}\text { Urban flood modeling with porous shallow-water } \\
\text { equations: A case study of model errors in the presence of } \\
\text { anisotropic porosity }\end{array}$ & \\
\hline 23 & $\begin{array}{l}\text { Guinot V. } \\
\text { et.al }\end{array}$ & $\begin{array}{l}\text { Advances in Water } \\
\text { Resources }\end{array}$ & 2017 & $\begin{array}{l}\text { Dual integral porosity shallow water model for urban flood } \\
\text { modelling. }\end{array}$ & \\
\hline 24 & $\begin{array}{l}\text { Bruwier M. } \\
\text { et.al }\end{array}$ & Journal of Hydrology & 2017 & $\begin{array}{l}\text { Shallow-water models with anisotropic porosity and } \\
\text { merging for flood modelling on Cartesian grids }\end{array}$ & \\
\hline 25 & $\begin{array}{l}\text { Glenis V. } \\
\text { et.al }\end{array}$ & $\begin{array}{l}\text { Environmental Modelling } \\
\text { and Software }\end{array}$ & 2018 & $\begin{array}{l}\text { A fully hydrodynamic urban flood modelling system } \\
\text { representing buildings, green space and interventions }\end{array}$ & \\
\hline 26 & $\begin{array}{l}\text { Ferrari A. } \\
\text { et.al }\end{array}$ & $\begin{array}{l}\text { Advances in water } \\
\text { resources }\end{array}$ & 2019 & $\begin{array}{l}\text { Flood inundation modeling in urbanized areas: A mesh- } \\
\text { independent porosity approach with anisotropic friction. }\end{array}$ & \\
\hline 27 & Xia X. et.al & $\begin{array}{l}\text { Advances in Water } \\
\text { Resources }\end{array}$ & 2019 & $\begin{array}{l}\text { A full-scale fluvial flood modelling framework based on a } \\
\text { high-performance integrated hydrodynamic modelling } \\
\text { system (HiPIMS). }\end{array}$ & \\
\hline 28 & $\begin{array}{l}\text { Seyoum S. } \\
\text { D. et.al }\end{array}$ & $\begin{array}{l}\text { Journal of Hydraulic } \\
\text { Engineering }\end{array}$ & 2011 & $\begin{array}{l}\text { Coupled 1D and Noninertia 2D Flood Inundation Model } \\
\text { for Simulation of Urban Flooding }\end{array}$ & \multirow{6}{*}{$\begin{array}{l}\text { Drainage network coupled to urban } \\
\text { surface model (Overlap with the } \\
\text { above does not repeat) }\end{array}$} \\
\hline 29 & $\begin{array}{l}\text { Bazin P. H. } \\
\text { et.al }\end{array}$ & $\begin{array}{l}\text { Journal of Hydraulic } \\
\text { Engineering }\end{array}$ & 2014 & $\begin{array}{l}\text { Modeling flow exchanges between a street and an } \\
\text { underground drainage pipe during urban floods }\end{array}$ & \\
\hline 30 & Chen Y. et.al & Environmental Research & 2015 & Urban flood risk warning under rapid urbanization & \\
\hline 31 & $\begin{array}{l}\text { Leandro J. } \\
\text { et.al }\end{array}$ & $\begin{array}{l}\text { Water Science and } \\
\text { Technology }\end{array}$ & 2016 & $\begin{array}{l}\text { A methodology for linking 2D overland flow models with } \\
\text { the sewer network model SWMM } 5.1 \text { based on dynamic } \\
\text { link libraries }\end{array}$ & \\
\hline 32 & $\begin{array}{l}\text { Martins R. } \\
\text { et.al }\end{array}$ & $\begin{array}{l}\text { Journal of } \\
\text { Hydroinformatics }\end{array}$ & 2017 & $\begin{array}{l}\text { A comparison of three dual drainage models: Shallow } \\
\text { water vs local inertial vs diffusive wave }\end{array}$ & \\
\hline 33 & Li Q. et.al & $\begin{array}{l}\text { Advances in Water } \\
\text { Resources, } 2020,137 .\end{array}$ & 2020 & $\begin{array}{l}\text { A novel 1D-2D coupled model for hydrodynamic } \\
\text { simulation of flows in drainage networks }\end{array}$ & \\
\hline 34 & $\begin{array}{l}\text { Yanqing L. } \\
\text { et.al }\end{array}$ & Journal of Hydrology & 2007 & $\begin{array}{l}\text { Coupling of hydrologic and hydraulic models for the } \\
\text { Illinois River Basin }\end{array}$ & \multirow{2}{*}{$\begin{array}{l}\text { Hydrological coupled to } \\
\text { hydrodynamic urban flood model }\end{array}$} \\
\hline 35 & $\begin{array}{l}\text { Bonnifait L. } \\
\text { et.al }\end{array}$ & $\begin{array}{l}\text { Advances in Water } \\
\text { Resources }\end{array}$ & 2009 & $\begin{array}{l}\text { Distributed hydrologic and hydraulic modelling with radar } \\
\text { rainfall input: Reconstruction of the 8-9 September } 2002 \\
\text { catastrophic flood event in the Gard region, France }\end{array}$ & \\
\hline
\end{tabular}




\begin{tabular}{|c|c|c|c|c|c|}
\hline 36 & $\begin{array}{l}\text { Mejia A.I. } \\
\text { et.al }\end{array}$ & Journal of Hydrology & 2011 & $\begin{array}{l}\text { Evaluating the effects of parameterized cross section } \\
\text { shapes and simplified routing with a coupled distributed } \\
\text { hydrologic and hydraulic model }\end{array}$ & \multirow{7}{*}{ Hydrogeomorphic approaches } \\
\hline 37 & Kim J. et.al & $\begin{array}{l}\text { Advances in Water } \\
\text { Resources }\end{array}$ & 2012 & $\begin{array}{l}\text { Coupled modeling of hydrologic and hydrodynamic } \\
\text { processes including overland and channel flow }\end{array}$ & \\
\hline 38 & $\begin{array}{l}\text { Zhanyan L. } \\
\text { et.al }\end{array}$ & Hydrology Research & 2019 & $\begin{array}{l}\text { A coupled hydrological and hydrodynamic model for flood } \\
\text { simulation }\end{array}$ & \\
\hline 39 & Rajib A. et.al & Journal of Hydrology & 2020 & $\begin{array}{l}\text { Towards a large-scale locally relevant flood inundation } \\
\text { modeling framework using SWAT and LISFLOOD-FP }\end{array}$ & \\
\hline 40 & $\begin{array}{l}\text { Nardi, F. } \\
\text { et.al }\end{array}$ & Irrigation and Drainage & 2013 & $\begin{array}{l}\text { Comparing a large-scale DEM-based floodplain } \\
\text { delineation algorithm with standard flood maps: The Tiber } \\
\text { River Basin case study }\end{array}$ & \\
\hline 41 & $\begin{array}{l}\text { Nardi, F. } \\
\text { et.al }\end{array}$ & $\begin{array}{l}\text { River Research and } \\
\text { Applications }\end{array}$ & 2018 & $\begin{array}{l}\text { Hydrologic scaling for hydrogeomorphic } \\
\text { mapping: Insights into human-induced floodplain } \\
\text { disconnectivity }\end{array}$ & \\
\hline 42 & Di B, G. et.al & $\begin{array}{l}\text { Natural Hazards and } \\
\text { Earth System Sciences }\end{array}$ & 2020 & $\begin{array}{l}\text { Brief communication: Comparing hydrological and } \\
\text { hydrogeomorphic paradigms for global flood hazard } \\
\text { mapping }\end{array}$ & \\
\hline 43 & $\begin{array}{l}\text { Dottori F. } \\
\text { et.al }\end{array}$ & $\begin{array}{l}\text { Physics and Chemistry of } \\
\text { the Earth }\end{array}$ & 2011 & $\begin{array}{l}\text { Developments of a flood inundation model based on the } \\
\text { cellular automata approach: testing different methods to } \\
\text { improve model performance. }\end{array}$ & \multirow{6}{*}{ Other methods } \\
\hline 44 & $\begin{array}{l}\text { Ghimire B. } \\
\text { et.al }\end{array}$ & $\begin{array}{l}\text { Journal of } \\
\text { Hydroinformatics }\end{array}$ & 2013 & $\begin{array}{l}\text { Formulation of a fast } 2 \mathrm{D} \text { urban pluvial flood model using } \\
\text { a cellular automata approach. }\end{array}$ & \\
\hline 45 & $\begin{array}{l}\text { Guidolin M. } \\
\text { et.al }\end{array}$ & $\begin{array}{l}\text { Environmental Modelling } \\
\text { and Software }\end{array}$ & 2016 & $\begin{array}{l}\text { A weighted cellular automata 2D inundation model for } \\
\text { rapid flood analysis. }\end{array}$ & \\
\hline 46 & $\begin{array}{l}\text { Yaseen ZM. } \\
\text { et.al }\end{array}$ & Journal of Hydrology & 2015 & $\begin{array}{l}\text { Artificial intelligence based models for stream-flow } \\
\text { forecasting: } 2000-2015 \text {. }\end{array}$ & \\
\hline 47 & $\begin{array}{l}\text { Wolfs V. } \\
\text { et.al }\end{array}$ & $\begin{array}{l}\text { Environmental Modelling } \\
\& \text { Software }\end{array}$ & 2014 & $\begin{array}{l}\text { Development of discharge-stage curves affected by } \\
\text { hysteresis using time varying models, model trees and } \\
\text { neural networks. }\end{array}$ & \\
\hline 48 & $\begin{array}{l}\text { Bermudez } \\
\text { M. et.al }\end{array}$ & $\begin{array}{l}\text { Water Resources } \\
\text { Management }\end{array}$ & 2018 & $\begin{array}{l}\text { Development and Comparison of Two Fast Surrogate } \\
\text { Models for Urban Pluvial Flood Simulations. }\end{array}$ & \\
\hline
\end{tabular}

